\title{
Adiponectin and adiponectin receptors in insulin resistance, diabetes, and the metabolic syndrome
}

\author{
Takashi Kadowaki,1,2 Toshimasa Yamauchi,1,3 Naoto Kubota,1,2,3 \\ Kazuo Hara,1,2 Kohjiro Ueki,1 and Kazuyuki Tobe ${ }^{1}$
}

\begin{abstract}
1Department of Metabolic Diseases, Graduate School of Medicine, University of Tokyo, Tokyo, Japan. 2Division of Applied Nutrition, National Institute of Health and Nutrition, Tokyo, Japan. ${ }^{3}$ Department of Integrated Molecular Science on Metabolic Diseases, Graduate School of Medicine, University of Tokyo, Tokyo, Japan.
\end{abstract}

Adiponectin is an adipokine that is specifically and abundantly expressed in adipose tissue and directly sensitizes the body to insulin. Hypoadiponectinemia, caused by interactions of genetic factors such as SNPs in the Adiponectin gene and environmental factors causing obesity, appears to play an important causal role in insulin resistance, type 2 diabetes, and the metabolic syndrome, which are linked to obesity. The adiponectin receptors, AdipoR1 and AdipoR2, which mediate the antidiabetic metabolic actions of adiponectin, have been cloned and are downregulated in obesity-linked insulin resistance. Upregulation of adiponectin is a partial cause of the insulin-sensitizing and antidiabetic actions of thiazolidinediones. Therefore, adiponectin and adiponectin receptors represent potential versatile therapeutic targets to combat obesity-linked diseases characterized by insulin resistance. This Review describes the pathophysiology of adiponectin and adiponectin receptors in insulin resistance, diabetes, and the metabolic syndrome.

The prevalence of obesity has increased dramatically in recent years $(1,2)$. It is commonly associated with type 2 diabetes, coronary artery disease, and hypertension, and the coexistence of these diseases has been termed the metabolic syndrome (3-7). Insulin resistance is a key feature of these diseases and is defined as a state that requires more insulin to obtain the biological effects achieved by a lower amount of insulin in the normal state. Thus, any defects in the insulin signaling cascade can cause insulin resistance. Insulin stimulates a signaling network composed of a number of molecules, initiating the activation of insulin receptor tyrosine kinase and phosphorylation of the insulin receptor substrate (IRS) proteins (e.g., IRS-1 and IRS-2) (8). Among several components of the network, the signaling axis of IRS proteins and PI3K, which activates downstream serine/threonine kinases including Akt, regulates most of the metabolic actions of insulin, such as suppression of hepatic glucose production and activation of glucose transport in muscle and adipocytes (9). It is known that this pathway is impaired at the multiple steps through alterations in the protein levels and activities of the signaling molecules, enzymes, and transcription factors in insulin resistance caused by obesity, a state of increased adiposity (9).

White adipose tissue (WAT) is a major site of energy storage and is important for energy homeostasis: it stores energy in the form of triglycerides during nutritional abundance and releases it as FFAs during nutritional deprivation $(10,11)$. While WAT provides a survival advantage in times of starvation, excess WAT is now linked to obesity-related health problems in the current nutritionally rich environment. Regulated by multiple hormonal signals, nuclear hormone receptors $(12,13)$, and the CNS (14),

Nonstandard abbreviations used: AdipoR, adiponectin receptor; AMPK, AMP-activated protein kinase; APPL, adaptor protein containing pleckstrin homology domain, phosphotyrosine-binding domain, and leucine zipper motif; GPCR, G protein-coupled receptor; HMW, high molecular weight; IRS, insulin receptor substrate; LMW, low molecular weight; MMW, middle molecular weight; PR-5, pathogenesis related-5; TZD, thiazolidinedione; WAT, white adipose tissue.

Conflict of interest: The authors have declared that no conflict of interest exists. Citation for this article: J. Clin. Invest. 116:1784-1792 (2006). doi:10.1172/JCI29126
WAT has been increasingly recognized as an important endocrine organ that secretes a number of biologically active "adipokines" (15-19). Some of these adipokines have been shown to directly or indirectly affect insulin sensitivity through modulation of insulin signaling and the molecules involved in glucose and lipid metabolism (20). Of these adipokines, adiponectin has recently attracted much attention because of its antidiabetic and antiatherogenic effects and is expected to be a novel therapeutic tool for diabetes and the metabolic syndrome (21). Indeed, a decrease in the circulating levels of adiponectin by genetic and environmental factors has been shown to contribute to the development of diabetes and the metabolic syndrome. The thiazolidinedione (TZD) class of antidiabetic drugs, which also have pleiotropic effects on cardiovascular diseases and lipid metabolism, is known to exert its effects partly through increasing the levels of the active form of adiponectin, as described below.

In this Review, we describe recent progress in research on the pathophysiological role of adiponectin and adiponectin receptors in insulin resistance, type 2 diabetes, and the metabolic syndrome. Since the length of this Review is limited, we recommend that readers also consult other recent reviews on adiponectin research (21-23).

\section{Association of hypoadiponectinemia with insulin resistance, diabetes, and the metabolic syndrome} Adiponectin, also termed Acrp30 (24), AdipoQ (25), apM1 (26), or GBP28 (27), was originally identified independently by 4 groups using different approaches. The Adiponectin gene encodes a secreted protein expressed exclusively in both WAT and brown adipose tissue. Adiponectin has a carboxyl-terminal globular domain and an amino-terminal collagen domain and is structurally similar to complement $1 \mathrm{q}(28,29)$, which belongs to a family of proteins that form characteristic multimers $(30,31)$. Adiponectin exists in a wide range of multimer complexes in plasma and combines via its collagen domain to create 3 major oligomeric forms: a low-molecular weight (LMW) trimer, a middle-molecular weight (MMW) hexamer, and high-molecular weight (HMW) 12- to 18-mer adiponectin $(32,33)$. In contrast to the expression of adipokines such 


\section{Table 1}

Physiological and pathophysiological conditions and treatment modalities associated with either decrease or increase in plasma adiponectin levels

\begin{tabular}{lc} 
Pathophysiological conditions or treatment modality & Ref. \\
Hypoadiponectinemia is associated with: & \\
Genetic variation in Adiponectin gene & $33,72-77$ \\
Obesity & $35-37$ \\
Insulin resistance & 37,38 \\
Type 2 diabetes & $37,39-44$ \\
Metabolic syndrome & 45 \\
Dyslipidemia & 45 \\
Cardiovascular disease & 46,47 \\
Hypertension & 48,49 \\
Sex hormones (androgen, testosterone) & 51,52 \\
Oxidative stress & 57 \\
Carbohydrate-rich diet & 56 \\
Increase in adiponectin levels is observed following: & \\
Administration of: & \\
TZDs & $58,79,104-108$ \\
Angiotensin II receptor blocker (ARB) & 110 \\
Angiotensin-converting enzyme inhibitors (ACEls) & 111 \\
Heart failure & 114 \\
Renal failure & 115 \\
Weight loss & 112,113 \\
Dietary factors: & \\
Soy protein & 53 \\
Oils & 54,55 \\
\hline
\end{tabular}

as TNF- $\alpha$ and resistin, which cause insulin resistance, adiponectin expression is reduced in obese, insulin-resistant rodent models (25). Plasma adiponectin levels are also decreased in an obese rhesus monkey model that frequently develops type 2 diabetes (34). Importantly, a decrease in plasma adiponectin levels preceded the onset of diabetes in these animals, in parallel with the observation of decreased insulin sensitivity (34). Plasma adiponectin levels have also been reported to be reduced in obese humans, particularly those with visceral obesity, and to correlate inversely with insulin resistance (35-38) (Table 1). Prospective and longitudinal studies $(37,39-44)$ have shown that lower adiponectin levels are associated with a higher incidence of diabetes. Adiponectin, but not inflammatory markers such as C-reactive protein and IL-6, has been shown to be significantly related to the development of type 2 diabetes in Pima Indians (44). Hypoadiponectinemia has also been demonstrated to be independently associated with the metabolic syndrome - indeed, more strongly than are any other inflammatory markers (45). Reduced plasma adiponectin levels are also commonly observed in a variety of states frequently associated with insulin resistance, such as cardiovascular disease (46, 47) and hypertension $(48,49)$.

How is the level of plasma adiponectin physiologically regulated? There is a sexual dimorphism in the circulating levels of adiponectin. Indeed, female humans and rodents have higher plasma adiponectin levels than males, suggesting that sexual hormones regulate the production of adiponectin, although it is controversial how these hormones, such as estrogen and testosterone, are involved in the regulation of plasma adiponectin level (50-52). Nevertheless, this may partly account for the fact that females are more sensitive to insulin than males. Some dietary factors, such as soy protein (53), fish oils (54), and linoleic acid (55), are also suggested to increase plasma adiponectin levels, which is consistent with the fact that intake of these factors is thought to have a protective effect on the development of diabetes. On the other hand, a carbohydrate-rich diet appears to decrease plasma adiponectin level (56). Oxidative stress has also been suggested to inhibit the expression of adiponectin (57). Although the mechanism underlying this regulation is unclear, this may contribute to the decrease in plasma adiponectin in obesity, which is associated with increased oxidative stress in adipose tissue. Thus, the plasma adiponectin level is affected by multiple factors, including gender, aging, and lifestyle.

\section{Discovery of the insulin-sensitizing action of adiponectin}

The insulin-sensitizing effect of adiponectin was first identified by 3 independent groups in 2001 (58-60). We assessed whether adiponectin was able to improve insulin resistance in $\mathrm{KKA}^{\mathrm{y}}$ mice (KK mice overexpressing the agouti protein), as a model of the metabolic syndrome and type 2 diabetes linked to obesity. Plasma adiponectin levels were decreased in KKAy mice fed a high-fat diet. Replenishment of adiponectin significantly ameliorated high-fat diet-induced insulin resistance and hypertriglyceridemia, which led us to propose that adiponectin is an insulin-sensitizing adipokine (58). These data also strongly suggested that the high-fat diet-induced, obesity-linked decrease in adiponectin level is causally involved in obesity-linked insulin resistance and the metabolic syndrome. Scherer and colleagues reported that an acute increase in the level of circulating adiponectin triggers a transient decrease in basal glucose level by inhibiting both the expression of hepatic gluconeogenic enzymes and the rate of endogenous glucose production in both wild-type and type 2 diabetic mice, and they proposed that adiponectin sensitizes the body to insulin (59). A truncated form of adiponectin that includes the globular domain cleaved proteolytically from full-length adiponectin has been reported to exist in plasma, although in very small amounts (60). Lodish and colleagues reported that a proteolytic cleavage product of adiponectin, which structurally resembles globular adiponectin, increases fatty-acid oxidation in muscle, decreases plasma glucose, and causes weight loss in mice (60).

Subsequently, the chronic effects of adiponectin on insulin resistance in vivo were investigated by generation of adiponectin transgenic mice $(61,62)$ or adiponectin-deficient mice (63-66). Globular adiponectin transgenic $o b / o b$ mice showed partial amelioration of insulin resistance and diabetes (61). Full-length adiponectin transgenic mice showed suppression of insulin-mediated endogenous glucose production (62). Our adiponectin-deficient mice showed mild insulin resistance with glucose intolerance while on a standard diet (63). The adiponectin-deficient mice examined by Maeda et al. exhibited a near-normal insulin sensitivity when fed a standard laboratory diet but developed severe insulin resistance, especially in skeletal muscle, in as few as 2 weeks on a high-fat, high-sucrose diet (64). Ma et al. reported that adiponectin-deficient mice displayed increased fatty-acid oxidation in skeletal muscle but showed no effect on either insulin sensitivity or glucose tolerance whether on a standard or a high-fat diet (65). Scherer's group reported that adiponectin-deficient mice showed mild insulin resistance in the liver while on a standard diet (66), and their phenotype was very similar to those of our adiponectin-deficient mice. On the other hand, some discrepancies in phenotypes that 


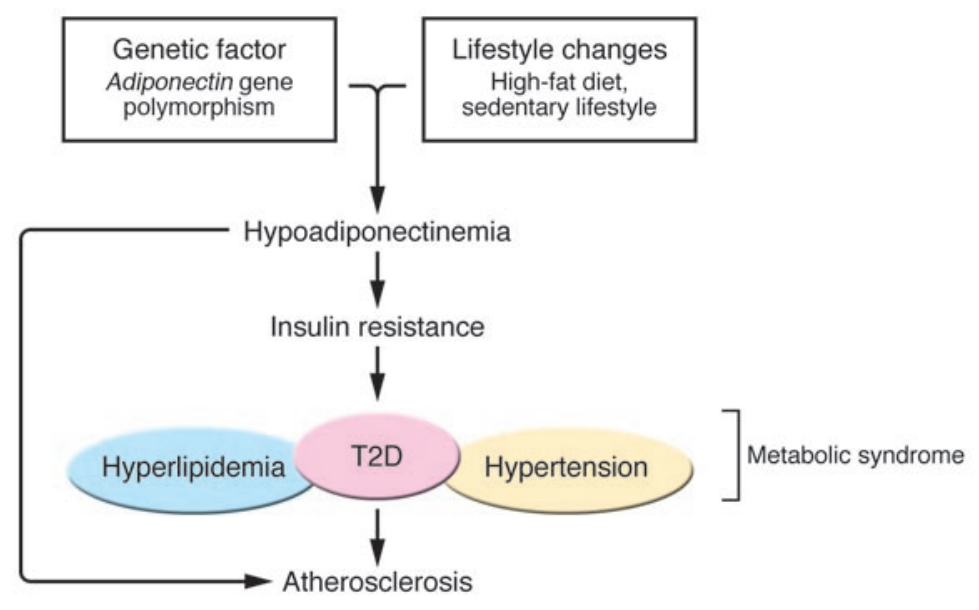

\section{Figure 1}

Adiponectin hypothesis for insulin resistance, the metabolic syndrome, and atherosclerosis. Reduced adiponectin levels can be caused by interactions of genetic factors such as SNP 276 in the Adiponectin gene itself and environmental factors, i.e., lifestyle changes that cause obesity, such as a high-fat diet and sedentary lifestyle. This reduction in adiponectin levels in turn appears to play an important causal role in the development of insulin resistance, type 2 diabetes (T2D), and metabolic disease, thereby indirectly causing atherosclerosis. Moreover, reduced adiponectin levels also directly play a causal role in the development of atherosclerosis. have been described among adiponectin-deficient mice are most likely due to differences in genetic background. Adiponectin-deficient mice exhibited other features of the metabolic syndrome, such as hyperlipidemia and hypertension $(48,63)$.

With respect to the molecular mechanisms underlying the insulin-sensitizing action of adiponectin, we found that full-length adiponectin stimulated AMP-activated protein kinase (AMPK) phosphorylation and activation in the liver, while globular adiponectin did so in both skeletal muscle and the liver (67). Blocking AMPK activation by use of a dominant-negative mutant inhibited these effects of full-length or globular adiponectin, indicating that stimulation of glucose utilization and fatty-acid combustion by adiponectin occurs through activation of AMPK (67). Thus, an adipocyte-derived antidiabetic hormone, adiponectin, activates AMPK, thereby directly regulating glucose metabolism and insulin sensitivity (67). These data also suggested that there may be 2 distinct receptors for adiponectin in the liver and skeletal muscle, with different binding affinities for globular and full-length adiponectin. Lodish, Ruderman, and colleagues also showed that the adiponectin globular domain could enhance muscle fat oxidation and glucose transport via AMPK activation and acetyl-CoA carboxylase inhibition (68). Scherer et al. reported that in adiponectin transgenic mice (62), reduced expression of gluconeogenic enzymes such as phosphoenolpyruvate carboxylase and glucose6-phosphatase is associated with elevated phosphorylation of hepatic AMPK, which may account for inhibition of endogenous glucose production by adiponectin $(59,67,69)$.

Adiponectin also increased fatty-acid combustion and energy consumption, in part via PPAR $\alpha$ activation, which led to decreased triglyceride content in the liver and skeletal muscle, and thereby a coordinated increase of in vivo insulin sensitivity (61).

\section{Adiponectin gene SNPs in human insulin resistance and type 2 diabetes}

The Adiponectin gene is located on chromosome $3 \mathrm{q} 27$, which has been reported to be linked to type 2 diabetes and the metabolic syndrome (70-72). Therefore, the Adiponectin gene appears to be a promising candidate susceptibility gene for type 2 diabetes. Among the SNPs in the Adiponectin gene, 1 SNP located $276 \mathrm{bp}$ downstream of the translational start site (SNP 276) was concomitantly associated with decreased plasma adiponectin level, greater insulin resistance, and an increased risk of type 2 diabetes (73). The subjects, both of whose 2 alleles of SNP 276 are the G (G/G genotype), had an approximately doubled risk for developing type 2 diabetes as compared with those with the T/T genotype (73). It is noteworthy that more than $40 \%$ of Japanese individuals have the "at-risk" G/G genotype, which makes subjects prone to genetically decreased adiponectin levels and thus susceptible to type 2 diabetes (73). Subjects with an I164T missense mutation in the globular domain of adiponectin had significantly lower plasma adiponectin levels than those without, independently of BMI (74).

Similar associations of the Adiponectin gene with susceptibility to type 2 diabetes have also been reported in other ethnic groups. In white German and North American subjects, SNP 276, either independently or as a haplotype together with SNP 45 in exon 2, was shown to be associated with obesity and insulin resistance $(75,76)$. In white French subjects, 2 SNPs in the promoter region of the Adiponectin gene, SNP 11377 and SNP 11391, were significantly associated with hypoadiponectinemia and type 2 diabetes (72). Taken together, these data strongly support the hypothesis that adiponectin plays a pivotal role in the pathogenesis of type 2 diabetes. A recent haplotype analysis based on a dense SNP map in a large sample clarified a 2-block linkage disequilibrium structure of the Adiponectin gene, the first block including the promoter SNPs and the second spanning the exons and introns (77). It is noteworthy that neither block has more than 1 SNP significantly associated with the plasma adiponectin level. The haplotypes in the first block were associated with increased adiponectin level, whereas the haplotypes in the second block were associated with decreased adiponectin level. This result indicated the existence of at least 2 causal haplotypes or SNPs in the Adiponectin gene.

Based on the significant body of evidence discussed above, we have proposed the "adiponectin hypothesis," in which reduced plasma adiponectin levels caused by interactions between genetic factors, such as SNPs in the Adiponectin gene itself, and environmental factors causing obesity, such as a sedentary lifestyle, may play a crucial role in the development of insulin resistance, type 2 diabetes, and the metabolic syndrome (21) (Figure 1).

\section{Role of HMW adiponectin in insulin resistance and type 2 diabetes}

Several observations support the hypothesis that HMW adiponectin is the more active form of the protein and has a more relevant role in insulin sensitivity and in protecting against diabetes. First, rare mutations - G84R and G90S - in the collagen domain are closely associated with type 2 diabetes $(33,73,78)$. Subjects with 


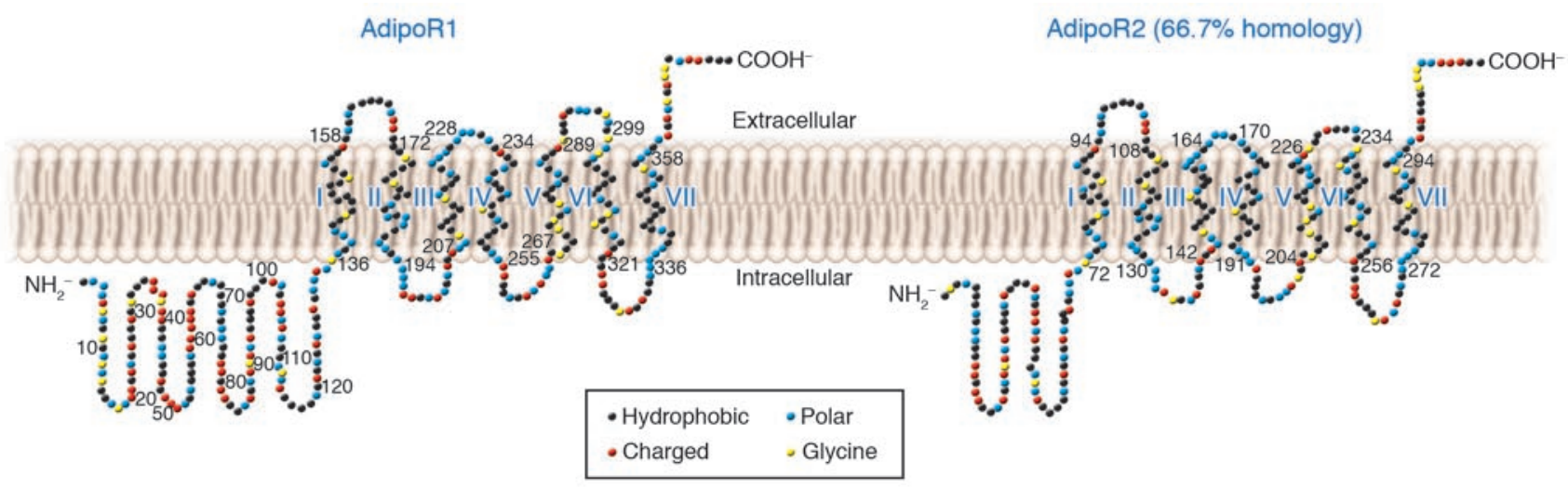

Figure 2

Structure of adiponectin receptors. AdipoR1 and AdipoR2 (66.7\% amino acid identity with AdipoR1) are predicted to contain 7 transmembrane domains but are structurally and topologically distinct from GPCRs. Redrawn with permission from Endocrine Reviews (21); copyright 2005, The Endocrine Society.

either of these 2 mutations have extremely low levels of HMW adiponectin (Table 1). Moreover, the 2 mutant adiponectins recombinantly expressed in NIH-3T3 fibroblasts were not able to form the HMW form of adiponectin. Second, increases in the ratio of plasma HMW adiponectin levels to total adiponectin levels correlate with improvement in insulin sensitivity during treatment with an insulin-sensitizing drug, TZD, in both mice and human diabetic patients, whereas increases in total serum adiponectin levels do not show good correlations with improvement in insulin sensitivity during treatment with TZD at the individual level (79). Third, the level of plasma HMW adiponectin was reported to be associated with parameters related to glucose homeostasis in a cohort study (80). It is noteworthy that the ratio of plasma HMW adiponectin to total adiponectin correlated more significantly with glucose and insulin levels than did the total adiponectin level (80), suggesting that alterations in plasma HMW adiponectin level may be more relevant to the prediction of insulin resistance than are total plasma adiponectin alterations. Consistent with this, levels of total adiponectin, HMW adiponectin, LMW adiponectin, and the HMW-to-total adiponectin ratio all correlated significantly with key features of central obesity and the insulin-stimulated glucose disposal rate (81). However, HMW adiponectin levels, not total adiponectin levels, are primarily responsible for these relationships, suggesting that measurement of the HMW adiponectin level may be superior to measurement of total adiponectin (81). Using an ELISA system for selective measurement of HMW adiponectin (82), we also found HMW adiponectin and the HMW-to-total adiponectin ratio to have significantly better power for the prediction of insulin resistance and the metabolic syndrome in humans (83). Thus, HMW adiponectin level may be the superior biomarker for insulin resistance, the metabolic syndrome, and type 2 diabetes.

\section{Cloning, function, and regulation of adiponectin receptors}

In order to further determine the pathophysiological significance and molecular mechanism of adiponectin action, we isolated cDNA for adiponectin receptors mediating the antidiabetic effects of adiponectin from a human skeletal muscle cDNA library by screening for globular adiponectin binding (84). The cDNA ana- lyzed encoded a protein designated human adiponectin receptor 1 (AdipoR1) (84). This protein is structurally conserved from yeast to humans (especially in the 7 transmembrane domains). Interestingly, the yeast homologue (YOL002c) plays a key role in metabolic pathways that regulate lipid metabolism, such as fatty-acid oxidation (85). Since at that time there may have been 2 distinct adiponectin receptors, as was described above (67), we searched for a homologous gene in the human and mouse databases. We found only 1 gene that was significantly homologous (67\% amino acid identity) with AdipoR1, which was termed AdipoR2 (84). AdipoR1 is ubiquitously expressed, including abundant expression in skeletal muscle, whereas AdipoR2 is most abundantly expressed in the mouse liver. AdipoR1 and AdipoR2 appear to be integral membrane proteins; the $\mathrm{N}$-terminus is internal and the C-terminus is external - opposite to the topology of all other reported G protein-coupled receptors (GPCRs) (84) (Figure 2). Expression of AdipoR1 and AdipoR2 or suppression of AdipoR1 and AdipoR2 expression supports our conclusion that AdipoR1 and AdipoR2 serve as receptors for globular and full-length adiponectin and mediate increased AMPK, PPAR $\alpha$ ligand activities, fattyacid oxidation, and glucose uptake by adiponectin (Figure 3 ).

Lodish's group reported that T-cadherin was capable of binding adiponectin in $\mathrm{C} 2 \mathrm{C} 12$ myoblasts; however, T-cadherin was not expressed in hepatocytes or the liver (86), the most important target organ $(66,69,87)$. Moreover, T-cadherin by itself was thought to have no effect on adiponectin cellular signaling or function, since T-cadherin is without an intracellular domain. These data raised the possibility that T-cadherin may be one of the adiponectin-binding proteins.

Most recently, a 2-hybrid study revealed that the C-terminal extracellular domain of AdipoR1 interacted with adiponectin, whereas the $\mathrm{N}$-terminal cytoplasmic domain of AdipoR1 interacted with APPL (adaptor protein containing pleckstrin homology domain, phosphotyrosine-binding domain, and leucine zipper motif) (88). Moreover, interaction of APPL with AdipoR1 in mammalian cells was stimulated by adiponectin binding, and this interaction played important roles in adiponectin signaling and adiponectin-mediated downstream events such as lipid oxidation and glucose uptake. These data clearly indicated that adiponectin receptors directly interacted with adiponectin and mediated adiponectin effects. Furthermore, 


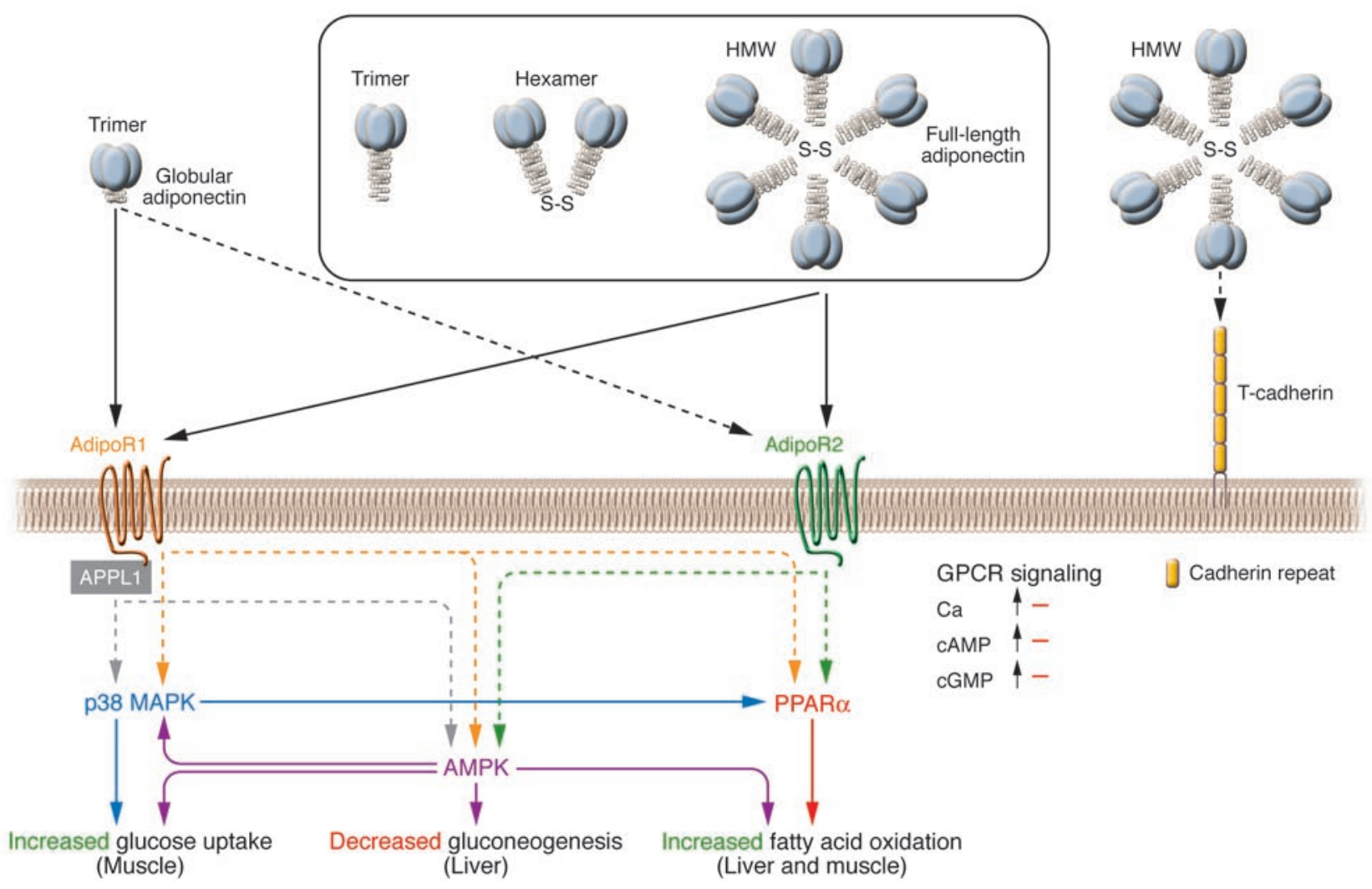

Figure 3

Signal transduction by adiponectin receptors. Globular adiponectin exists as a trimer, whereas full-length adiponectin exists as at least 3 species of multimers: an LMW trimer, an MMW hexamer, and an HMW multimer. Suppression of AdipoR1 by RNA interference markedly reduces globular adiponectin binding, whereas suppression of AdipoR2 by RNA interference largely reduces full-length adiponectin-specific binding (21, 84). The dotted line between AdipoR2 and globular adiponectin reflects that AdipoR2 is a relatively low-affinity receptor for globular adiponectin. AdipoR1 and AdipoR2 do not seem to be coupled with G proteins, since overexpression of AdipoR1/R2 has little effect on cAMP, cGMP, and intracellular calcium levels, but instead these receptors activate unique sets of signaling molecules such as PPAR $\alpha$, AMPK, and p38 MAPK. In C2C12 myocytes overexpressing AdipoR1/R2, adiponectin stimulates PPAR $\alpha$, AMPK, and p38 MAPK activation, glucose uptake, and fatty-acid oxidation (84). Suppression of AMPK or PPAR $\alpha$ partially reduces adiponectin-stimulated fatty-acid oxidation, and suppression of AMPK or p38 MAPK partially reduces adiponectin-stimulated glucose uptake. In hepatocytes overexpressing AdipoR1/R2, adiponectin stimulates PPAR $\alpha$ or AMPK and fattyacid oxidation (84). Suppression of AMPK or PPAR $\alpha$ in these hepatocytes partially reduces adiponectin-stimulated fatty-acid oxidation. Moreover, treatment with adiponectin reduces plasma glucose levels and molecules involved in gluconeogenesis in the liver, and dominant-negative AMPK partly reduces these effects. These data support the conclusion that AdipoR1 and AdipoR2 serve as receptors for globular and full-length adiponectin and mediate increased AMPK, PPAR $\alpha$ ligand activities, p38 MAPK, and adiponectin-induced biological functions. T-cadherin is capable of binding adiponectin but is thought to have no effect on adiponectin cellular signaling, since T-cadherin lacks an intracellular domain (86). Interaction of APPL1 (adaptor protein containing pleckstrin homology domain, phosphotyrosine-binding domain, and leucine zipper motif 1) with AdipoR1 appears to play important roles in adiponectin signaling and adiponectin-mediated downstream events such as lipid oxidation and glucose uptake (88). S-S, disulfide bond. Adapted with permission from Endocrine Reviews (21); copyright 2005, The Endocrine Society.

these data strongly supported that the $\mathrm{N}$-terminus of adiponectin receptors is internal and the C-terminus is external (88).

The expression levels of both AdipoR1 and AdipoR2 were significantly decreased in muscle and adipose tissue of insulin-resistant $o b / o b$ mice, probably in part because of obesity-linked hyperinsulinemia (89). Moreover, adiponectin-induced activation of AMPK was impaired in the skeletal muscle of $o b / o b$ mice. These data suggest that adiponectin resistance is present in $o b / o b$ mice, presumably due to decreased expression of AdipoR1 and AdipoR2 (89). Thus, obesity decreases not only plasma adiponectin levels but also AdipoR1/R2 expression, thereby reducing adiponectin sensitivity and leading to insulin resistance, which in turn aggravates hyperinsulinemia, creating a "vicious cycle" (89). Adiponectin receptor expression in the skeletal muscle of type 2 diabetic patients has been reported to be decreased (90). In addition, a correlation has been reported between adiponectin receptor gene expression and insulin sensitivity in nondiabetic Mexican Americans with or without a family history of type 2 diabetes (91). Moreover, AdipoR1 mRNA expression was positively correlated with in vivo insulin and C-peptide concentrations, firstphase insulin secretion, and plasma triglyceride and cholesterol concentrations before and after adjustment for sex, age, waist-to-hip ratio, and body fat. Expression of AdipoR2 mRNA was clearly associated only with plasma triglyceride concentrations. In multivariate linear regression models, mRNA expression of AdipoR1, but not AdipoR2, was a determinant of first-phase insulin secretion independently of insulin sensitivity and body fat (92). Since AdipoR1 and AdipoR 2 are expressed in pancreatic $\beta$ cells, these receptors may play a role in insulin secretion (93). 


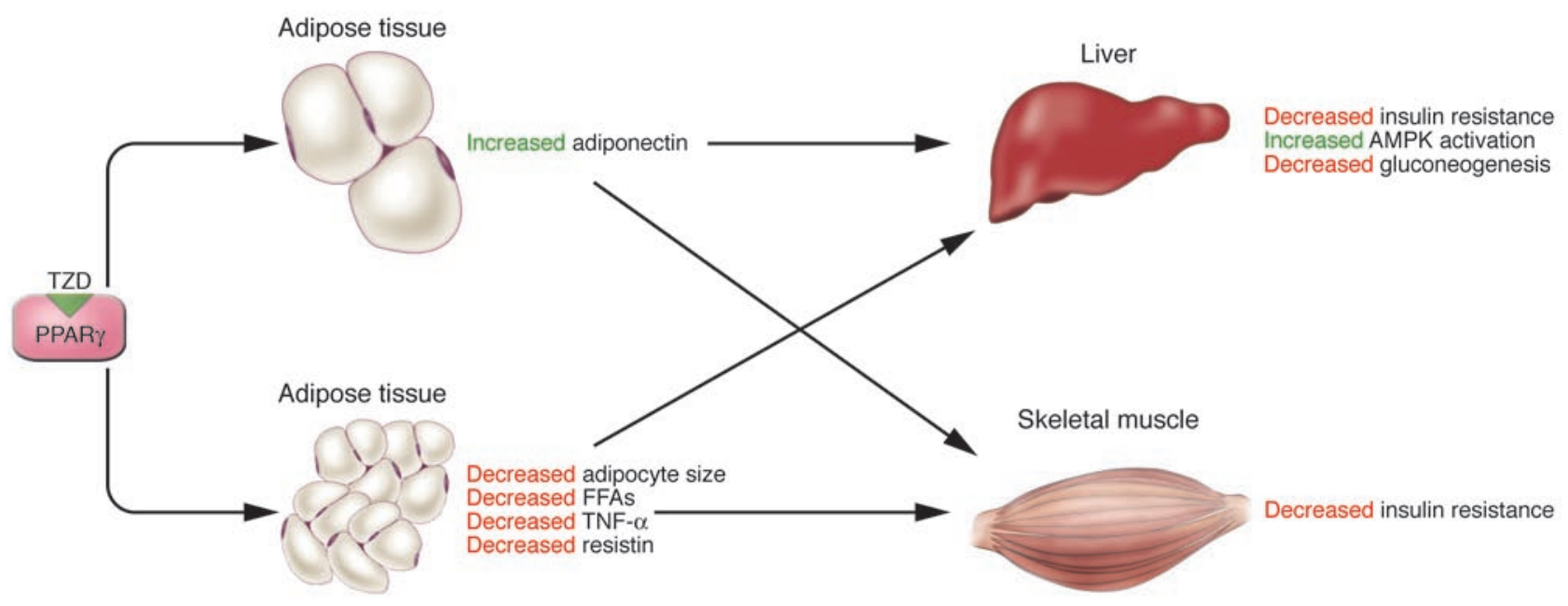

Figure 4

TZDs ameliorate insulin resistance and diabetes by both adiponectin-dependent and -independent pathways. We propose that there are 2 different pathways in the amelioration of insulin resistance induced by the PPAR $\gamma$ agonists TZDs, such as pioglitazone and probably rosiglitazone. One involves an adiponectin-dependent pathway and the other an adiponectin-independent pathway. TZDs increase adiponectin levels, ameliorating insulin resistance, increasing AMPK activation, and decreasing gluconeogenesis in the liver. On the other hand, independently of adiponectin, TZDs decrease adipocyte size, serum FFA levels, and expression of TNF- $\alpha$ and resistin, thus contributing to amelioration of insulin resistance in skeletal muscle.

\section{Adiponectin and adiponectin receptors as therapeutic targets}

According to our adiponectin hypothesis (21), a therapeutic strategy for the treatment of insulin resistance, type 2 diabetes, the metabolic syndrome, and cardiovascular disease may include the upregulation of plasma adiponectin levels, the upregulation of adiponectin receptors, or the development of adiponectin receptor agonists.

TZD-mediated upregulation of plasma adiponectin level. TZDs are known to improve systemic insulin sensitivity in animal models of obesity-linked insulin resistance and diabetes, by enhancing glucose disposal in skeletal muscle and suppressing gluconeogenesis in the liver. TZDs have been widely used as therapeutic agents for the treatment of type 2 diabetes (94-99). TZDs have been proposed to ameliorate insulin resistance by binding to and activating PPAR $\gamma$ in adipose tissue, thereby promoting adipocyte differentiation and increasing the number of small adipocytes that are more sensitive to insulin (100-103). Plasma adiponectin levels have been shown to be upregulated by TZDs $(58,79,104-108)$ (Table 1), and HMW adiponectin is a predominant form of adiponectin upregulated by TZDs (89). TZDs may upregulate adiponectin by generating small adipocytes that abundantly express and secrete adiponectin $(100,102,109)$ and/or directly activating Adiponectin gene transcription (106). TZDs may also directly facilitate the generation of HMW adiponectin. Since adiponectin is an insulin-sensitizing adipokine, it is reasonable to speculate that the action whereby TZDs increase insulin sensitivity is mediated, at least in part, by increased plasma adiponectin levels. However, whether the TZD-induced increase in plasma adiponectin level is causally involved in TZD-mediated insulin-sensitizing effects has not been addressed experimentally. Adiponectin-deficient (Adipo-/-) ob/ob mice with a C57BL/6 background were used to investigate whether the PPAR $\gamma$ agonist pioglitazone is capable of ameliorating insulin resistance in the absence of adiponectin (63, 87). The absence of adiponectin had no effect on either the obesi- ty or the diabetic phenotype of these mice. The severity of insulin resistance and diabetes observed in $o b / o b$ mice was significantly reduced in association with significant upregulation of serum adiponectin levels by low-dose $(10 \mathrm{mg} / \mathrm{kg})$ pioglitazone treatment. Amelioration of insulin resistance in $o b / o b$ mice was attributed to decreased glucose production and increased AMPK levels in the liver, but not to increased glucose uptake in skeletal muscle. In contrast, the severity of insulin resistance and diabetes was not reduced in $A$ dipo $^{-/-}$ob/ob mice (87). With high-dose pioglitazone treatment, the insulin resistance and diabetes of $o b / o b$ mice were again significantly ameliorated; this was attributed not only to decreased glucose production in the liver but also to increased glucose uptake in skeletal muscle. Interestingly, Adipo-/-ob/ob mice also displayed significant amelioration of insulin resistance and diabetes. The serum FFA and triglyceride levels as well as adipocyte sizes in $o b / o b$ and $A d i p o^{-/-} o b / o b$ mice were unchanged after low-dose pioglitazone treatment but were significantly reduced to a similar degree after high-dose pioglitazone treatment. Moreover, the expression of TNF- $\alpha$ and resistin in adipose tissues of $o b / o b$ and $A d i p o^{-/-o b / o b}$ mice were unchanged after low-dose pioglitazone but were decreased after high-dose pioglitazone. Although both high and low doses of pioglitazone ameliorated insulin resistance and diabetes, the underlying mechanisms may be different (87). We propose that there are 2 different pathways in the amelioration of insulin resistance induced by TZDs such as pioglitazone, and probably rosiglitazone. One involves an adiponectin-dependent pathway and the other an adiponectin-independent pathway (Figure 4). TZDs increase adiponectin levels via activation of Adiponectin gene transcription without stimulating adipocyte differentiation $(58,106)$, thereby increasing AMPK activation, decreasing gluconeogenesis in the liver, and ameliorating insulin resistance and type 2 diabetes. On the other hand, independently of adiponectin, TZDs induce adipocyte differentiation, leading to an increase in the number of small adipocytes, which is 
associated with decreased serum FFA levels and decreased TNF- $\alpha$ and resistin expression, together contributing to amelioration of insulin resistance in skeletal muscle (87).

Scherer's group demonstrated that $o b / o b$ mice showed significantly improved glucose tolerance after rosiglitazone treatment, whereas Adipo-/-ob/ob mice responded only partially to this treatment and remained severely glucose intolerant (66), suggesting that rosiglitazone ameliorated glucose intolerance via both adiponectin-dependent and -independent pathways. Moreover, rosiglitazone significantly increased AMPK activity in the livers of wildtype mice, whereas it had no effect on $A_{d i p o^{-/-}}$mice. In skeletal muscle, AMPK activity was also significantly increased in wildtype mice, while no increase was detectable in Adipo-/- mice. These data are in complete agreement with our data. Other pharmacological agents as well as lifestyle changes have also been reported to be associated with upregulation of plasma adiponectin levels $(53,55,110-115)$ (Table 1).

Upregulation of adiponectin receptors and development of adiponectin receptor agonists. Since AdipoR1 and AdipoR2 are downregulated in obesity-linked insulin resistance and diabetes, both upregulation of AdipoR1 and AdipoR2 expression and agonism of AdipoR1 and AdipoR2 may be a logical approach to providing a novel treatment modality for insulin resistance and type 2 diabetes $(84,89)$. Previously, Staels's group reported that adiponectin receptors are expressed in human macrophages and that adiponectin receptor expression levels may be regulated by agonists of the nuclear receptors PPAR $\alpha, \operatorname{PPAR} \gamma$, and liver X receptor (116). We have recently shown that, in $\mathrm{KKA}^{\mathrm{y}}$ mice, a PPAR $\alpha$ agonist reversed decreases in AdipoR1 and AdipoR2 expression, which was lower in white and brown adipose tissue of KKAy mice than in that of wild-type control KK mice (117). These data suggested that dual activation of PPAR $\gamma$ and PPAR $\alpha$ enhanced the action of adiponectin by increasing both total and HMW adiponectin level and adiponectin receptor number, which can ameliorate obesitylinked insulin resistance.

Osmotin is a member of the pathogenesis related-5 (PR-5) family of plant defense proteins ( 24 members in Arabidopsis thaliana) that induce apoptosis in yeast. It is ubiquitous in fruits and vegetables, etc., and the genes encoding the PR-5 protein sequenced from many different species are about 50-95\% identical. PR-5 family proteins are also extremely stable and may remain active even when in contact with the human digestive or respiratory system. Bressan's group isolated a yeast clone that exhibited hypersensitivity to osmotin, sequenced the cDNA inserts, and found that PHO36/YOL002c, the yeast homologue of AdipoR1, is a receptor for osmotin (118). X-ray crystallographic studies revealed that both globular adiponectin and osmotin consist of antiparallel $\beta$-strands arranged in the shape of a $\beta$-barrel. Domain I (lectinlike domain) of osmotin showed similarity to globular adiponectin in $3 \mathrm{D}$ structure, suggesting that these 2 proteins share the lectinlike domain (118). Interestingly, osmotin activates AMPK via adiponectin receptors in mammalian $\mathrm{C} 2 \mathrm{C} 12$ myocytes (118). These data raise the possibility that further research examining similarities in adiponectin and osmotin may facilitate the development of potential adiponectin receptor agonists (118). Although further studies will be needed to determine the physiological and pathophysiological roles of AdipoR1 and AdipoR2, the enhancement or mimicking of adiponectin action through modulation of expression and/or function of AdipoR1 and AdipoR2 can be a novel therapeutic strategy for the treatment of insulin resistance, the metabolic syndrome, and type 2 diabetes.

In summary, adiponectin is an adipokine that exerts a potent insulin-sensitizing effect by binding to its receptors such as AdipoR1 and AdipoR2, leading to activation of AMPK, PPAR $\alpha$, and presumably some other unknown signaling pathways. Indeed, circulating levels of adiponectin, especially HMW adiponectin, are positively correlated with insulin sensitivity and altered by various genetic and environmental factors, pathological conditions, and medications. Thus, monitoring the levels of HMW adiponectin is a good predictable marker for type 2 diabetes and the metabolic syndrome. Moreover, methods to increase adiponectin levels, such as TZD administration, are expected to be effective for the treatment of these diseases. In the future, enhancing or mimicking adiponectin action through modulation of expression and/or function of the adiponectin receptors may be a novel and promising therapeutic strategy for insulin resistance, type 2 diabetes, and the metabolic syndrome.

\section{Acknowledgments}

This work was supported by the Program for Promotion of Fundamental Studies in Health Sciences of the Organization for Pharmaceutical Safety and Research of Japan, a grant from the Human Science Foundation (to T. Kadowaki), a Grant-in-Aid for the Development of Innovative Technology from the Ministry of Education, Culture, Sports, Science, and Technology of Japan (to T. Kadowaki), a Grant-in Aid for Creative Scientific Research (10NP0201) from the Japan Society for the Promotion of Science (to T. Kadowaki), and by Health Science Research Grants (Research on Human Genome and Gene Therapy) from the Ministry of Health, Labour and Welfare of Japan (to T. Kadowaki).

Address correspondence to: Takashi Kadowaki, Department of Metabolic Diseases, Graduate School of Medicine, University of Tokyo, 7-3-1 Hongo, Bunkyo-ku, Tokyo 113-8655, Japan. Phone: 81-3-5800-8818; Fax: 81-3-5800-9797; E-mail: kadowaki-3im@ h.u-tokyo.ac.jp.
1. Flier, J.S. 2004. Obesity wars: molecular progress confronts an expanding epidemic. Cell. 116:337-350.

2. Friedman,J.M. 2000. Obesity in the new millennium. Nature. 404:632-634.

3. Reaven, G.M. 1998. Role of insulin resistance in human disease. Diabetes. 37:1595-1607.

4. Kaplan, N.M. 1989. The deadly quartet. Upper-body obesity, glucose intolerance, hypertriglyceridemia, and hypertension. Arch. Intern. Med. 149:1514-1520.

5. Matsuzawa, Y. 1997. Pathophysiology and molecular mechanisms of visceral fat syndrome: the Japanese experience. Diabetes Metab. Rev. 13:3-13.

6. National Cholesterol Education Program. 2001. Executive Summary of the Third Report of the
National Cholesterol Education Program (NCEP) Expert Panel on Detection, Evaluation, and Treatment of High Blood Cholesterol in Adults (Adult Treatment Panel III). JAMA. 285:2486-2497.

7. International Diabetes Federation. 2005. A new worldwide definition of the metabolic syndrome. http:// www.idf.org/home/index.cfm? unode=32EF2063B966-468F-928C-A5682A4E3910.

8. Taniguchi, C.M., Emanuelli, B., and Kahn, C.R. 2006. Critical nodes in signaling pathways: insights into insulin action. Nat. Rev. Mol. Cell Biol. 7:85-96.

9. Saltiel, A.R., and Kahn, C.R. 2001. Insulin signaling and the regulation of glucose and lipid metabolism. Nature. 414:799-806.
10. Kahn, C.R. 2000. Triglycerides and toggling the tummy. Nat. Genet. 25:6-7.

11. Spiegelman, B.M., and Flier, J.S. 2001. Obesity and regulation of energy balance. Cell. 104:531-543.

12. Lowell, B.B. 1999. PPARgamma: an essential regulator of adipogenesis and modulator of fat cell function. Cell. 99:239-242.

13. Chawla, A., Saez, E., and Evans, R.M. 2000. Don't know much bile-ology. Cell. 103:1-4.

14. Obici, S., Feng, Z., Arduini, A., Conti, R., and Rossetti, L. 2003. Inhibition of hypothalamic carnitine palmitoyltransferase- 1 decreases food intake and glucose production. Nat. Med. 9:756-761.

15. Hotamisligil, G.S., Shargill, N.S., and Spiegelman, B.M. 
1993. Adipose expression of tumor necrosis factoralpha: direct role in obesity-linked insulin resistance. Science. 259:87-91.

16. Zhang, Y., et al. 1994. Positional cloning of the mouse obese gene and its human homologue. Nature. 372:425-432.

17. Steppan, C.M., et al. 2001. The hormone resistin links obesity to diabetes. Nature. 409:307-312.

18. Yang, Q., et al. 2005. Serum retinal binding protein 4 contributes to insulin resistance in obesity and type 2 diabetes. Nature. 436:356-362.

19. Lazar, M.A. 2006. The humoral side of insulin resistance. Nat. Med. 12:43-44.

20. Kershaw, E.E., and Flier, J.S. 2004. Adipose tissue as an endocrine organ. J. Clin. Endocrinol. Metab. 89:2548-2556

21. Kadowaki, T., and Yamauchi, T. 2005. Adiponectin and adiponectin receptors. Endocr. Rev. 26:439-451.

22. Berg, A.H., and Scherer, P.E. 2005. Adipose tissue, inflammation, and cardiovascular disease. Circ. Res. 96:939-949.

23. Okamoto, Y., Kihara, S., Funahashi, T., Matsuzawa, Y., and Libby, P. 2006. Adiponectin: a key adipocytokine in metabolic syndrome. Clin. Sci. (Lond.) 110:267-278.

24. Scherer, P.E., Williams, S., Fogliano, M., Baldini, G., and Lodish, H.F. 1995. A novel serum protein similar to C1q, produced exclusively in adipocytes. J. Biol. Chem. 270:26746-26749.

25. Hu, E., Liang, P., and Spiegelman, B.M. 1996. AdipoQ is a novel adipose-specific gene dysregulated in obesity. J. Biol. Chem. 271:10697-10703.

26. Maeda, K., et al. 1996. cDNA cloning and expression of a novel adipose specific collagen-like factor, apM1 (AdiPose Most abundant Gene transcript 1). Biochem. Biophys. Res. Commun. 221:286-289.

27. Nakano, Y., Tobe, T., Choi-Miura, N.H., Mazda, T., and Tomita, M. 1996. Isolation and characterization of GBP28, a novel gelatin-binding protein purified from human plasma. J. Biochem. (Tokyo). 120:803-812

28. Shapiro, L., and Scherer, P.E. 1998. The crystal structure of a complement-1q family protein suggests an evolutionary link to tumor necrosis factor. Curr. Biol. 8:335-338.

29. Yokota, T., et al. 2000. Adiponectin, a new member of the family of soluble defense collagens, negatively regulates the growth of myelomonocytic progenitors and the functions of macrophages. Blood. 96:1723-1732.

30. Crouch, E., Persson, A., Chang, D., and Heuser, J. 1994. Molecular structure of pulmonary surfactant protein D (SP-D). J. Biol. Chem. 269:17311-17319.

31. McCormack, F.X., et al. 1997. The Cys6 intermolecular disulfide bond and the collagen-like region of rat SP-A play critical roles in interactions with alveolar type II cells and surfactant lipids. J. Biol. Chem. 272:27971-27979.

32. Pajvani, U.B., et al. 2003. Structure-function studies of the adipocyte-secreted hormone Acrp30/adiponectin. Implications for metabolic regulation and bioactivity. J. Biol. Chem. 278:9073-9085.

33. Waki, H., et al. 2003. Impaired multimerization of human adiponectin mutants associated with diabetes: molecular structure and multimer formation of adiponectin. J. Biol. Chem. 278:40352-40363.

34. Hotta, K., et al. 2001. Circulating concentrations of the adipocyte protein adiponectin are decreased in parallel with reduced insulin sensitivity during the progression to type 2 diabetes in rhesus monkeys. Diabetes. 50:1126-1133.

35. Arita, Y., et al. 1999. Paradoxical decrease of an adipose-specific protein, adiponectin, in obesity. Biochem. Biophys. Res. Commun. 257:79-83.

36. Ryo, M., et al. 2004. Adiponectin as a biomarker of the metabolic syndrome. Circ. J. 68:975-981.

37. Yatagai, T., et al. 2003. Hypoadiponectinemia is associated with visceral fat accumulation and insu- lin resistance in Japanese men with type 2 diabetes mellitus. Metabolism. 52:1274-1278.

38. Yamamoto, Y., Hirose, H., Saito, I., Nishikai, K., and Saruta, T. 2004. Adiponectin, an adipocytederived protein, predicts future insulin-resistance: two-year follow-up study in Japanese population. J. Clin. Endocrinol. Metab. 89:87-90.

39. Lindsay, R.S., et al. 2002. Adiponectin and development of type 2 diabetes in the Pima Indian population. Lancet. 360:57-58.

40. Daimon, M., et al. 2003. Decreased serum levels of adiponectin are a risk factor for the progression to type 2 diabetes in the Japanese population: the Funagata study. Diabetes Care. 26:2015-2020.

41. Snehalatha, C. et al. 2003. Plasma adiponectin is an independent predictor of type 2 diabetes in Asian Indians. Diabetes Care. 26:3226-3229.

42. Spranger, J., et al. 2003. Adiponectin and protection against type 2 diabetes mellitus. Lancet. 361:226-228

43. Duncan, B.B., et al. 2004. Adiponectin and the development of type 2 diabetes: the Atherosclerosis Risk in Communities Study. Diabetes. 53:2473-2478.

44. Krakoff, J., et al. 2003. Inflammatory markers, adiponectin, and risk of type 2 diabetes in the Pima Indian. Diabetes Care. 26:1745-1751.

45. Matsushita, K., et al. 2006. Comparison of circulating adiponectin and proinflammatory markers regarding their association with metabolic syndrome in Japanese men. Arterioscler. Thromb. Vasc. Biol. 26:871-876

46. Kumada, M., et al. 2003. Association of hypoadiponectinemia with coronary artery disease in men. Arterioscler. Thromb. Vasc. Biol. 23:85-89.

47. Pischon, T., et al. 2004. Plasma adiponectin levels and risk of myocardial infarction in men. JAMA. 291:1730-1737.

48. Ouchi, N., et al. 2003. Association of hypoadiponectinemia with impaired vasoreactivity. Hypertension. 42:231-234.

49. Adamczak, M., et al. 2003. Decreased plasma adiponectin concentration in patients with essential hypertension. Am. J. Hypertens. 16:72-75.

50. Combs, T.P., et al. 2003. Sexual differentiation, pregnancy, calorie restriction, and aging affect the adipocyte-specific secretory protein adiponectin. Diabetes. 52:268-276.

51. Nishizawa, H., et al. 2002. Androgens decrease plasma adiponectin, an insulin-sensitizing adipocyte-derived protein. Diabetes. 51:2734-2741.

52. Xu, A., et al. 2005. Testosterone selectively reduces the high molecular weight form of adiponectin by inhibiting its secretion from adipocytes. J. Biol. Chem. 280:18073-18080.

53. Nagasawa, A., et al. 2002. Effects of soy protein diet on the expression of adipose genes and plasma adiponectin. Horm. Metab. Res. 34:635-639.

54. Flachs, P., et al. 2006. Polyunsaturated fatty acids of marine origin induce adiponectin in mice fed a high-fat diet. Diabetologia. 49:394-397.

55. Nagao, K., et al. 2003. Conjugated linoleic acid enhances plasma adiponectin level and alleviates hyperinsulinemia and hypertension in Zucker diabetic fatty $(\mathrm{fa} / \mathrm{fa})$ rats. Biochem. Biophys. Res. Commun. 310:562-566

56. Pischon, T., et al. 2005. Association between dietary factors and plasma adiponectin concentrations in men. Am. J. Clin. Nutr. 81:780-786.

57. Furukawa, S., et al. 2004. Increased oxidative stress in obesity and its impact on metabolic syndrome. J. Clin. Invest. 114:1752-1761. doi:10.1172/JCI200421625.

58. Yamauchi, T., et al. 2001. The fat-derived hormone adiponectin reverses insulin resistance associated with both lipoatrophy and obesity. Nat. Med. 7:941-946

59. Berg, A.H., et al. 2001. The adipocyte-secreted protein Acrp30 enhances hepatic insulin action. Nat. Med. 7:947-953.
60. Fruebis, J., et al. 2001. Proteolytic cleavage product of $30-\mathrm{kDa}$ adipocyte complement-related protein increases fatty acid oxidation in muscle and causes weight loss in mice. Proc. Natl. Acad. Sci. U. S. A. 98:2005-2010.

61. Yamauchi, T., et al. 2003. Globular adiponectin protected ob/ob mice from diabetes and ApoEdeficient mice from atherosclerosis. J. Biol. Chem. 278:2461-2468

62. Combs, T.P., et al. 2004. A transgenic mouse with a deletion in the collagenous domain of adiponectin displays elevated circulating adiponectin and improved insulin sensitivity. Endocrinology. 145:367-383.

63. Kubota, N., et al. 2002. Disruption of adiponectin causes insulin resistance and neointimal formation. J. Biol. Chem. 277:25863-25866.

64. Maeda, N., et al. 2002. Diet-induced insulin resistance in mice lacking adiponectin/ACRP30. Nat. Med. 8:731-737.

65. Ma, K., et al. 2002. Increased beta-oxidation but no insulin resistance or glucose intolerance in mice lacking adiponectin. J. Biol. Chem. 277:34658-34661.

66. Nawrocki, A.R., et al. 2006. Mice lacking adiponectin show decreased hepatic insulin sensitivity and reduced responsiveness to peroxisome proliferatoractivated receptor gamma agonists. J. Biol. Chem. 281:2654-2660.

67. Yamauchi, T., et al. 2002. Adiponectin stimulates glucose utilization and fatty-acid oxidation by activating AMP-activated protein kinase. Nat. Med. 8:1288-1295.

68. Tomas, E., et al. 2002. Enhanced muscle fat oxidation and glucose transport by ACRP30 globular domain: acetyl-CoA carboxylase inhibition and AMP-activated protein kinase activation. Proc. Natl. Acad. Sci. U. S. A. 99:16309-16313.

69. Combs, T.P., et al. 2001. Endogenous glucose production is inhibited by the adipose-derived protein Acrp30. J. Clin. Invest. 108:1875-1881. doi:10.1172/ JCI200114120.

70. Kissebah, A., et al. 2000. Quantitative trait loci on chromosomes 3 and 17 influence phenotypes of the metabolic syndrome. Proc. Natl. Acad. Sci.U. S. A. 97:14478-14483.

71. Mori, Y., et al. 2002. Genome-wide search for type 2 diabetes in Japanese affected sib-pairs confirms susceptibility genes on $3 q, 15 q$, and $20 q$ and identifies two new candidate loci on $7 p$ and $11 p$. Diabetes. 51:1247-1255.

72. Vasseur, F., et al. 2002. Single-nucleotide polymorphism haplotypes in the both proximal promoter and exon 3 of the APM1 gene modulate adipocytesecreted adiponectin hormone levels and contribute to the genetic risk for type 2 diabetes in French Caucasians. Hum. Mol. Genet. 11:2607-2614.

73. Hara, K., et al. 2002. Genetic variation in the gene encoding adiponectin is associated with an increased risk of type 2 diabetes in the Japanese population. Diabetes. 51:536-540.

74. Kondo, H., et al. 2002. Association of adiponectin mutation with type 2 diabetes: a candidate gene for the insulin resistance syndrome. Diabetes. 51:2325-2328.

75. Stumvoll, M., et al. 2002. Association of the T-G polymorphism in adiponectin (exon 2) with obesity and insulin sensitivity: interaction with family history of type 2 diabetes. Diabetes. 51:37-41.

76. Menzaghi, C., et al. 2002. A haplotype at the adiponectin locus is associated with obesity and other features of the insulin resistance syndrome. Diabetes. 51:2306-2312.

77. Woo, J.G., et al. 2006. Interactions between noncontiguous haplotypes in the adiponectin gene ACDC are associated with plasma adiponectin. Diabetes. 55:523-529.

78. Tasanen, K., et al. 2000. Collagen XVII is destabilized by a glycine substitution mutation in the cell adhesion 
domain Col15. J. Biol. Chem. 275:3093-3099.

79. Pajvani, U.B., et al. 2004. Complex distribution, not absolute amount of adiponectin, correlates with thiazolidinedione-mediated improvement in insulin sensitivity. J. Biol. Chem. 279:12152-12162.

80. Lara-Castro, C., et al. 2006. Adiponectin multimeric complexes and the metabolic syndrome trait cluster. Diabetes. 55:249-259.

81. Fisher, F.F., et al. 2005. Serum high molecular weight complex of adiponectin correlates better with glucose tolerance than total serum adiponectin in Indo-Asian males. Diabetologia. 48:1084-1087.

82. Ebinuma, H., et al. 2006. A novel ELISA system for selective measurement of human adiponectin multimers by using proteases. Clin. Chim. Acta. doi:10.1016/j.cca.2006.03.014

83. Hara, K., et al. 2006. Measurement of the highmolecular weight form of adiponectin in plasma is useful for the prediction of insulin resistance and metabolic syndrome. Diabetes Care. 29:1357-1362.

84. Yamauchi, T., et al. 2003. Cloning of adiponectin receptors that mediate antidiabetic metabolic effects. Nature. 423:762-769.

85. Karpichev, I.V., et al. 2002. Multiple regulatory roles of a novel Saccharomyces cerevisiae protein, encoded by YOL002c, in lipid and phosphate metabolism. J. Biol. Chem. 277:19609-19617.

86. Hug, C., et al. 2004. T-cadherin is a receptor for hexameric and high-molecular-weight forms of Acrp30/adiponectin. Proc. Natl. Acad. Sci. U. S. A. 101:10308-10313.

87. Kubota, N., et al. 2006. Pioglitazone ameliorates insulin resistance and diabetes by both adiponectin-dependent and -independent pathways. J. Biol. Chem. 281:8748-8755.

88. Mao, X., et al. 2006. APPL1 binds to adiponectin receptors and mediates adiponectin signalling and function. Nat. Cell Biol. 8:516-523.

89. Tsuchida, A., et al. 2004. Insulin/Foxo1 pathway regulates expression levels of adiponectin receptors and adiponectin sensitivity. J. Biol. Chem. 279:30817-30822.

90. Civitarese, A.E., et al. 2004. Adiponectin receptors gene expression and insulin sensitivity in non-diabetic Mexican Americans with or without a family history of type 2 diabetes. Diabetologia. 47:816-820.

91. Debard, C., et al. 2004. Expression of key genes of fatty acid oxidation, including adiponectin receptors, in skeletal muscle of type 2 diabetic patients.
Diabetologia. 47:917-925.

92. Harald, S., et al. 2004. Expression of adiponectin receptor mRNA in human skeletal muscle cells is related to in vivo parameters of glucose and lipid metabolism. Diabetes. 53:2195-2201.

93. Kharroubi, I., et al. 2003. Expression of adiponectin receptors in pancreatic beta cells. Biochem. Biophys. Res. Commun. 312:1118-1122.

94. Bowen, L., et al. 1991. The effect of CP 68,722 , a thiozolidinedione derivative, on insulin sensitivity in lean and obese Zucker rats. Metabolism. 40:1025-1030

95. Nolan, J.J., et al. 1994. Improvement in glucose tolerance and insulin resistance in obese subjects treated with troglitazone. N. Engl. J. Med. 331:1188-1193.

96. Saltiel, A.R. 2001. New perspectives into the molecular pathogenesis and treatment of type 2 diabetes. Cell. 104:517-529.

97. Mauvais-Jarvis, F., et al. 2000. Understanding the pathogenesis and treatment of insulin resistance and type 2 diabetes mellitus: what can we learn from transgenic and knockout mice? Diabetes Metab. 26:433-448.

98. Yki-Jarvinen, H. 2004. Thiazolidinediones. N. Engl. J. Med. 351:1106-1118.

99. Rangwala, S.M., et al. 2004. Peroxisome proliferator-activated receptor gamma in diabetes and metabolism. Trends Pharmacol. Sci. 25:331-336.

100.Okuno, A., et al. 1998. Troglitazone increases the number of small adipocytes without the change of white adipose tissue mass in obese Zucker rats. J. Clin. Invest. 101:1354-1361.

101.Evans, R.M., et al. 2004. PPARs and the complex journey to obesity. Nat. Med. 10:355-361.

102.Kubota, N., et al. 1999. PPAR gamma mediates high-fat diet-induced adipocyte hypertrophy and insulin resistance. Mol. Cell. 4:597-609.

103. Olefsky, J.M., et al. 2000. PPAR gamma and the treatment of insulin resistance. Trends Endocrinol. Metab. 11:362-368.

104.Yu, J.G., et al. 2002. The effect of thiazolidinediones on plasma adiponectin levels in normal, obese, and type 2 diabetic subjects. Diabetes. 51:2968-2974.

105.Chandran, M., et al. 2003. Adiponectin: more than just another fat cell hormone? Diabetes Care. 26:2442-2450.

106.Iwaki, M., et al. 2003. Induction of adiponectin, a fat-derived antidiabetic and antiatherogenic factor by nuclear receptors. Diabetes. 52:1655-1663.

107. Hirose, H., et al. 2002. Effects of pioglitazone on metabolic parameters, body fat distribution, and serum adiponectin levels in Japanese male patients with type 2 diabetes. Metabolism. 51:314-317.

108. Maeda, N., et al. 2001. PPAR $\gamma$ ligands increase expression and plasma concentrations of adiponectin, an adipose-derived protein. Diabetes. 50:2094-2099.

109. Yamauchi, T et al 2001. The mechanisms by which both heterozygous peroxisome proliferator-activated receptor gamma (PPARgamma) deficiency and PPARgamma agonist improve insulin resistance. J. Biol. Chem. 276:41245-41254.

110.Furuhashi, M., et al. 2003. Blockade of the reninangiotensin system increases adiponectin concentrations in patients with essential hypertension. Hypertension. 42:76-81.

111.Koh, K.K., et al. 2005. Vascular and metabolic effects of combined therapy with ramipril and simvastatin in patients with type 2 diabetes. Hypertension. 45:1088-1093.

112.Yang, W.S., et al. 2001. Weight reduction increases plasma levels of an adipose-derived anti-inflammatory protein, adiponectin. J. Clin. Endocrinol. Metab. 86:3815-3819.

113.Esposito, K., et al. 2003. Effect of weight loss and lifestyle changes on vascular inflammatory markers in obese women: a randomized trial. JAMA. 289:1799-1804.

114.Kistorp, C., et al. 2005. Plasma adiponectin, body mass index, and mortality in patients with chronic heart failure. Circulation. 112:1756-1762.

115. Tentolouris, N., et al. 2004. Plasma adiponectin concentrations in patients with chronic renal failure: relationship with metabolic risk factors and ischemic heart disease. Horm. Metab. Res. 36:721-727.

116. Chinetti, G., et al. 2004. Expression of adiponectin receptors in human macrophages and regulation by agonists of the nuclear receptors PPARalpha, PPARgamma, and LXR. Biochem. Biophys. Res. Commun. 314:151-158.

117. Tsuchida, A., et al. 2005. Peroxisome proliferatoractivated receptor (PPAR) alpha activation increases adiponectin receptors and reduces obesity-related inflammation in adipose tissue: comparison of activation of PPARalpha, PPARgamma, and their combination. Diabetes. 54:3358-3370.

118. Narasimhan, M.L., et al. 2005. Osmotin is a homolog of mammalian adiponectin and controls apoptosis in yeast through a homolog of mammalian adiponectin receptor. Mol. Cell. 17:171-180. 